\title{
Der Anteil der Mediziner an der Frühphase der Planktonforschung
}

\author{
Von Rüdiger Porep, Kiel
}

Meeresbiologie war um die Mitte des 19. Jahrhunderts en vogue und blieb es fast ein halbes Jahrhundert lang. Besonders mit der Meeresfauna beschäftigte sich eine Unzahl von Forschern (während die Flora im Vergleich dazu tatsächlich überaus arm an Formen ist). Man müßte unter den Anatomen und sonst naturwissenschaftlich ausgerichteten Medizinern dieser Zeit, der Blütezeit der vergleichenden Anatomie, geradezu suchen, wollte man einen finden, der sich nicht in irgendeiner Form mit der Meeresfauna befaßt hat. Wie lebhaft das Interesse an diesem Forschungszweig war, mögen nur zwei Beispiele illustrieren. Es verging kaum eine der «Versammlungen Deutscher Naturforscher und Ärzte ${ }^{1}$, auf der nicht meeresbiologische Themen von Medizinern angesprochen wurden ${ }^{2}$. Oder greifen wir einen Band aus dem Archiv für Anatomie, Physiologie und wissenschaftliche Medicin, kurz Müllers Archiv, z.B. ganz willkürlich den von 1850, heraus, so finden wir von vierzig Publikationen fünfzehn mit Themen aus der Meereszoologie überschrieben ${ }^{3}$.

Die eben genannten Publikationsorgane verraten schon, daß es reizvoll wäre, den Anteil der Mediziner an der Meeresbiologie abzugrenzen, doch läßt die erdrückende Fülle an Material keine andere Wahl, als ein ganz spezielles Gebiet herauszugreifen. So soll hier versucht werden, den Beitrag der Mediziner an der Planktonforschung zu skizzieren.

Unter Plankton werden alle im Wasser freischwebenden Lebewesen verstanden, deren Eigenbeweglichkeit geringer ist als die des Wassers. Dieser Begriff, der heute von jedem auch nur etwas naturwissenschaftlich Bewanderten verstanden und richtig gedeutet wird, ganz im Gegensatz zu dem Pendant Nekton (alle freibeweglichen Wasserbewohner, deren Eigenbeweglichkeit größer ist als die des Wassers) oder auch zum Benthos (alle nicht schwimmenden Wasserbewohner), ist noch recht jung. Victor Hensen, auf

1 Siehe Amtl. Bericht Vers. Dtsch. Naturforscher u. Ärzte, 7.-50. Versammlung, 1828-1877; und Verh. Ges. Dtsch. Naturforscher u. Ärzte, 63.-85. Versammlung, 1890-1913.

2 Brigitte Hoppe, Meeresbiologie in den ersten hundert Jahren der Versammlungen Deutscher Naturforscher und Ärzte. Medizinhistorisches Journal 1 (1966) 31-42.

3 Arch.Anat. Physiol. wiss. Med.1850. 
den noch ausführlich zurückzukommen sein wird, gebraucht ihn zum erstenmal im Jahre 1887 in seiner in Fachkreisen so berühmt gewordenen Schrift Über die Bestimmung des Plankton's oder des im Meere treibenden Materials an Pflanzen und Thieren ${ }^{4}$. Als Hensen für den Begriffsinhalt «das Treibende», der die zu benennende Materie am besten traf, einen prägnanten, auch für fremde Zungen sprechbaren Namen suchte, wandte er sich an den Kieler Professor für Klassische Philologie und Beredsamkeit Richard Förster (1843-1922), der sich vom Griechischen inspirieren ließ und fand, daß das von $\pi \lambda \alpha v a ́ \omega=$ ich treibe abgeleitete Wort Plankton allen Wünschen gerecht werde ${ }^{5}$. Schon in seiner Rektoratsrede am 5.März 1887 konnte Hensen die homerische Poesie als Quelle der Idee für diese Namensgebung anführen, wo Odysseus $\mu \alpha \dot{\lambda} \alpha$ o $\pi \lambda \lambda \dot{\alpha} \pi \lambda \dot{\alpha} \gamma \chi \vartheta \eta$, gar viel umhergetrieben wird $^{6,7}$.

Einige Leitlinien sollen das Zurechtfinden in der Geschichte der Planktonforschung erleichtern. Sehr brauchbar ist für diese Zwecke das Vier-Perioden-Schema von STIASNY ${ }^{8}$, an das ich mich im folgenden lose anlehnen will.

Die erste Periode ist die der Vorarbeiten für die systematische Planktonforschung. Sie reicht vom Altertum bis in die Mitte der vierziger Jahre des 19. Jahrhunderts und ist charakterisiert durch vom Zufall abhängige Forschungen über Bau, Lebensweise, Entwicklung und systematische Stellung einzelner Planktonformen. Zufällig sind diese Erkenntnisse deshalb, weil nur solche Spezies erfaßt wurden, die durch ihre Größe oder ihr massenhaftes Auftreten die Aufmerksamkeit auf sich lenkten, zudem leicht zu fangen waren, was meist durch einfaches Herausschöpfen mit beliebigen Gefäßen geschah.

Aus dieser Periode seien nur zwei Mediziner genannt. Der eine ist Chri-

4 Victor Hensen, Über die Bestimmung des Plankton's oder des im Meere treibenden Materials an Pflanzen und Thieren. Fünfter Bericht der Kommission zur wissenschaftlichen Untersuchung der deutschen Meere in Kiel für die Jahre 1882 bis 1886, XII.-XVI. Jahrgang, S. 1-107, Berlin 1887.

5 ib., S.1.

6 Victor Hensen, Die Naturwissenschaft im Universitätsverband, Kiel 1887.

7 Homer, Odyssee I, Vers $1 / 2$.

${ }^{8}$ Gustay Stiasny, Das Plankton des Meeres, Sammlung Göschen Nr. 675, Berlin/Leipzig 1913, S.16-18. 
Stian Gottfried Ehrenberg (1795-1876), eine schon zu Lebzeiten sehr umstrittene Persönlichkeit. Seit dem Jahre 1847 war Ehrenberg Inhaber eines Ordinariats für Geschichte der Heilkunde in Berlin, ist in diesem Fach jedoch weder als Forscher noch als Lehrer jemals tätig geworden. Er beschäftigte sich mit kleinsten Lebewesen, z. B. Infusorien ${ }^{9}$. So vorbereitet, war er es, der das seit Menschengedenken bekannte Phänomen des Meeresleuchtens aufklären konnte, indem er es richtig als von mikroskopischen Meerestierchen verursacht deutete ${ }^{10}$, von deren Existenz man bislang noch nichts gewußt hatte. Allerdings war es seinem Ruhme abträglich, daß er den Einzellern alle Organe zuschrieb, die auch die höheren Tiere besitzen. Diese Behauptung fordertez. B. Koellikers Unwillen heraus : «Wenn Herr Prof. Ehrenberg nicht Professor in Berlin und dortiges Mitglied der Akademie wäre, so würde man ihn schon längst der Geschichte (i.e. der Vergessenheit) anheimgegeben haben. Ich begreife nicht, wie man das, was man unter dem Mikroskop sieht, gerade so auf die allerwillkürlichste und unangemessenste Weise deuten kann!»11

Der Zufallsperiode der Planktonforschung gehört als zweiter Mediziner Johann Friedrich Eschscholtz (1793-1831) an. Gleich nach seiner Promotion zum Dr. med. im Jahre 1815 nahm er eine Stelle als Schiffsarzt auf dem russischen Forschungsschiff «Rurik» an und machte dessen Reise um die Welt mit. Ein Jahr nach seiner Rückkehr, nämlich 1819, erhielt er die Prosektur und eine außerordentliche Professur in Dorpat, war also Anatom, übernahm aber schon 1822 zusätzlich die Direktion des «zoologischen Cabinettes », also der zoologischen Sammlungen, und hielt Vorlesungen auch in Zoologie. Noch einmal, vom Juli 1823 bis August 1824, fungierte er als Schiffsarzt bei der Expedition der «Predprijatel».12 Die Früchte seiner zoologischen Forschungen auf diesen Expeditionen legte er in dem großen Werk nieder: Zoologischer Atlas, Abbildungen und Beschreibungen neuer Thierarten, während des Flottenkapitäns v. Kotzebue zweiter Reise beobachtet, Heft 1-4, ${ }^{13}$. Darin sind auch viele Tierarten, die heute zum Plankton gezählt werden, zum erstenmal beschrieben. Von 1828 an war EscHscholtz o. Professor für Anatomie in Dorpat ${ }^{12}$.

9 Biographisches Lexikon der hervorragenden Ärzte aller Zeiten und Völker, herausgegeben von August Hirsch, 3., unveränderte Auflage. München/Berlin 1962.

10 Christian Gottrfied Ehrenberg, Das Leuchten des Meeres, Berlin 1835.

11 Zit. nach Ernst HaeckeL, Briefe an die Eltern 1852-1856, Berlin 1921, S. 52.

12 Siehe Anm. 9.

13 Johann Friedrich Eschscholtz, Zoologischer Atlas etc., Heft 1-4, Berlin 1829-1831. 
Die zweite Periode der Planktonforschung ist gekennzeichnet durch die Entwicklung einer für Plankter angemessenen Fangtechnik, die dadurch möglich gewordene gezielte Suche nach neuen Arten, die nun zu Tausenden beschrieben werden, durch die Aufklärung der Entwicklungsgeschichte vieler Arten und, auf alledem fußend, durch die Entstehung einer Systematik der Plankter im Sinne Linnés. Auf diese Phase bezieht sich die Bemerkung am Anfang dieser Ausführungen, daß eine unübersehbare Zahl von Medizinern an der Planktonforschung mitgewirkt habe, weshalb hier nur die besten Köpfe und ihre Schulen berücksichtigt werden können. Allen voran, chronologisch und von der Bedeutung her gesehen, ist der Berliner Anatom und Physiologe Johannes MüLler (1801-1858) zu nennen. Im Jahre 1845 fuhr Joh. Müller zum erstenmal nach Helgoland, um Meerestiere zu beobachten. Er leitete damit eine Wallfahrt der Morphologen ans Meer ein, die sich über mehr als drei Jahrzehnte für die Meeresbiologie und damit für die Planktologie als unendlich fruchtbar erweisen sollte.

Der Pionier Joh. Müller, gemeinsam mit seinen Schülern WILMs ${ }^{14}$ und Busch $^{15}$, zu denen sich 1845 v. Franque gesellte, der 1846 von Wagner ${ }^{16}$ abgelöst wurde, schöpfte zunächst noch einfach Meerwasser in ein Gefäß, um Untersuchungsmaterial zu gewinnen. Die anschließende mikroskopische Durchmusterung brachte immer wieder neue Arten kleinster, im Wasser schwebender Meereslebewesen an den $\mathrm{Tag}^{17}$.

Ganz entscheidend für die Suche nach neuen Arten war dann die Entwicklung einer ergiebigeren Fangtechnik. Es war wiederum Joh. Müller, der das Planktonnetz einführte. Diese für die Planktologie wahrhaft historische Stunde schlug, allen Monographien und Nachschlagewerken zufolge, im Jahre 1845, wobei regelmäßig die Angabe der Quelle vermißt wird. Nach meinen eigenen Untersuchungen spricht Joh. Müller bei den Unternehmun-

14 Robert Ferdinand Wilms (1824-1880) wurde Chirurg am Diakonissenkrankenhaus Bethanien in Berlin, ab 1862 dirigierender Arzt der chirurgischen Abteilung dort; er wurde durch seine chirurgischen Leistungen sehr bekannt.

15 Karl David Wilhelm Busch (1826-1881) veröffentlichte schon als Student mehrere Arbeiten über meeresbiologische Themen in Arch.Anat. Physiol. wiss. Med.1847 ff.; 1855 o. Professor der Chirurgie in Bonn.

16 Karl Ernst Albrecht Wagner (1827-1871) wurde 1858 o. Professor der Chirurgie in Königsberg.

17 Johannes Müller, Bericht über einige neue Thierformen der Nordsee,Arch.Anat. Physiol. wiss. Med.,1846, S.101-110. 
gen des Jahres 1845 nur von Schöpfen ${ }^{18,19}$, wohingegen in einem Brief an seinen Sohn Max aus dem Jahre 1846 zu lesen ist: "Wir haben mehrere Arten zu fischen ins Werk gesetzt, die wir im vorigen Jahr noch nicht hatten ...», ${ }^{20}$ während er an seine Frau Nanny schreibt: «... bald sieht man uns mit unsern kleinen Schmetterlingsnetzen zum Fischen gehen ». ${ }^{21}$

Wie sah nun die von Joh. Müller inaugurierte, so erfolgreiche neue Fangmethode aus ? Zwar gibt der Autor selbst eine knappe Schilderung ${ }^{22}$, doch eine weit plastischere Darstellung findet sich bei ERnst HaEgKel in einem Brief, den er 1860 aus Messina an seine Braut schrieb : «... Die Radiolarien [eine Ordnung der Zooplankter] sind sämtlich ausschließlich pelagische Tierchen, d.h. sie leben nur schwimmend auf der Oberfläche des tiefen Meeres, von der sie nur auf kurze Zeit schwinden, wenn heftige Wellenbewegungen und Sturm sie nötigt, sich in einige Tiefe herabzulassen. Dieser Umstand erleichtert ihren Fang sehr, ja, macht ihn eigentlich allein möglich. Man fischt sie nämlich von der Oberfläche, von der sie jeden Quadratfuß zu Hunderten bedecken, mittelst des feinen Mullnetzes weg, eine Methode, die zuerst von Johannes Müller mit dem größten Glück zum Fang aller pelagischen Tiere in weitestem Umfang angewandt wurde und welche die überraschendsten Blicke in eine ganz neue Welt reichsten tierischen Lebens eröffnet hat. Während die Barke durch schwachen Ruderschlag langsam fortbewegt wird, hält man das Netz beständig halb eingetaucht und filtriert so gleichsam eine große Menge Seewasser durch. Von Zeit zu Zeit wird dann das Netz herausgenommen, umgekehrt und der nach außen gewendete Innenteil ausgespült in dem mit Seewasser gefüllten Glas und Eimer, wo dann die in den Maschen hängengebliebenen feinsten Geschöpfchen wieder frei werden und zu Boden fallen. Dieser Bodensatz in den Gefäßen, von dem das überstehende geklärte Wasser nachher zu Haus abgegossen wird, ist nun eine ganz unerschöpfliche Quelle der reichsten und merkwürdigsten Naturgenüsse, indem er eine Unmasse der merkwürdigsten und interessan-

18 Siehe Anm. 17, S. 101.

19 Johannes MüLler, Fortsetzung des Berichtes über einige neue Thierformen der Nordsee, Arch. Anat. Physiol. wiss. Med. 1847, S.157-206.

20 Wilhelm HaberLing, Johannes Müller, das Leben des rheinischen Naturforschers, Band 9 der Reihe: Große Männer, Studien zur Biologie des Genies, herausgegeben von Wilhelm Ostwald, Leipzig 1924, S. 292.

21 ib., S. 295.

22 Johannes Müller, Über den allgemeinen Plan in der Entwickelung der Echinodermen, Abh. Kgl.Akad.Wiss. Berlin (Physik. Abh.) 1852, S. 26. 
testen Geschöpfchen, besonders aber Larven von aller Art, enthält, ja, zuweilen ganz allein daraus zusammengesetzt ist. Johannes Müller hat aus dieser herrlichen Fundgrube viele Jahre hindurch unendlichen Stoff zu den schönsten Untersuchungen geschöpft .... ${ }^{23}$.

Zum Aussehen eines solchen Müllerschen Planktonnetzes äußerten sich die Briten sehr prosaisch so: es sehe aus wie eine «lange Nachtmütze». ${ }^{24}$ Auf Joh. Müller geht die erste wissenschaftliche Bezeichnung alles dessen zurück, was sich auf die oben angegebene Weise fangen ließ. Er nannte es «pelagischen Auftrieb», wobei der Name «Auftrieb» von keinem Geringeren als JACов Grimm (1785-1863), dem Begründer der deutschen Sprachwissenschaft, vorgeschlagen wurde ${ }^{25}$.

Das Meer hatte Joh. Müller völlig gefangengenommen; immer wieder suchte er es auf, um seine Forschungen weiterzutreiben, worunter besonders die über die Metamorphose der Echinodermen herausragen, die ihn über zehn Jahre lang, bis zu seinem Tode, fesselten. Wie sehr Joh. Müller zum Meere strebte, mag eine Aufstellung seiner Forschungsreisen illustrieren: 1845 und 1846 Helgoland; 1847 Helsingör; 1848 Ostende; 1849 Marseille und, später im Jahr, Nizza; 1850-1852 insgesamt vier Reisen nach Triest; 1853 Sizilien, besonders Messina; 1854 Helgoland; 1855 Bergen; 1856 Nizza; 1857 St. Tropez ${ }^{26}$. Fügt man noch Neapel hinzu, so hat man eine Liste der wichtigsten Orte, die überhaupt besucht werden konnten. Auf fast jeder dieser Reisen wurde Joh. Müller von einigen Schülern begleitet, worunter in der Regel Studenten zu verstehen sind; doch ist keiner von diesen Planktologe geworden.

Auch die zweite herausragende Gestalt dieser Forschungsperiode, Albert voN Koelliker (1817-1905), Anatom in Würzburg, hatte bei seinen Reisen ans Mittelmeer immer eine Gruppe von Forschern um sich. So berichtete Koelliker im Jahre 1852 in einer Versammlung der Physico-Medica in Würzburg von einem Forschungsaufenthalt in Messina. Seine Begleiter waren Heinrich Müller (1820-1864), damals gerade zum a.o. Professor für topographische und vergleichende Anatomie ernannt, und der spätere Professor für Anatomie in Jena und Heidelberg Carl Gegenbaur (1826

23 Ernst Haecked, Italienfahrt. Briefe an die Braut 1859/60, Leipzig 1921, S.161-162.

24 John Murray and Johan HJort, The Depths of the Ocean, London 1912, S. 34.

25 Zit. nach Gottfried Koller, Das Leben des Biologen Johannes Müller 1801-1858, in der Reihe: Große Naturforscher, herausgegeben von HeInz Degen, Band 23, Stuttgart 1958, S.164.

${ }^{26}$ Siehe Anm. 25, S. 239-240. 
bis 1903) ${ }^{27}$. Gegenbaur kehrte 1852 nicht mit den beiden anderen Forschern nach Würzburg zurück, sondern hielt sich bis zum Frühjahr 1853 in Messina auf. Auch er hat für die Systematik einiger Tierstämme der Plankter erhebliches geleistet ${ }^{28}$.

Aus der großen Zahl der Mediziner, die über die vergleichende Anatomie zur Planktonforschung fanden, soll nur noch eine Persönlichkeit herausgehoben werden, an der sich der weitere Verlauf der Entwicklung der Wissenschaft sehr gut zeigen läßt: Ernst Haeckel (1834-1919). Haeckel versuchte von vornherein nicht mehr, Anatomie und Zoologie in sich zu vereinen, sondern er wandte sich, nachdem er den Dr. med. erworben hatte, ganz von der Medizin ab und derZoologie zu. Haeckel steht damit am Wendepunkt einer Entwicklung: künftig wird das Feld der Planktonsystematik ganz von Zoologen beherrscht. Haeckel war im Sommer 1854 nach Helgoland gereist, um meeresbiologische Studien zu treiben, und war dort auf Joh. Müller getroffen; seine Liebe zum Plankton war entbrannt ${ }^{29}$.

Im Sommer 1856 lud ihn dann Koelliker ein, ihn nach Nizza zu begleiten, worüber Haeckel sich vor Freude kaum zu lassen wußte ${ }^{30}$. Auch er ging dann, wie vor ihm schon Gegenbaur, im Oktober 1859 nach Messina und blieb dort bis zum 1.Mai $1860^{31}$. Die Früchte seiner Forschungsarbeiten legte er im Jahre 1862 in Form einer großen Radiolarienmonographie vor ${ }^{32}$.

Höhepunkt und Abschluß der zweiten Phase der Planktonforschung bildet die große britische «Challenger»-Expedition (1872-1876) unter Sir Wyville Thomson. An der Auswertung des gewaltigen Materials, die noch im 19. Jahrhundert abgeschlossen werden konnte, nahmen auch viele deutsche Forscher teil. Zu den Bearbeitern des Planktons gehören Koelliker und sein Schüler Haeckel ${ }^{33}$.

27 Zit. nach Anm. 11, S. 22.

${ }^{28}$ MaX FÜrbringer, Carl Gegenbaur, in Heidelberger Professoren aus dem 19. Jahrhundert etc., Bd. 2, S. 401 und S.455-458, Heidelberg 1903.

29 Siehe Anm. 11, S. 111-125.

30 Siehe Anm. 11, S. 202-209.

31 Johannes Hemleben, Ernst Haeckel in Selbstzeugnissen und Bilddokumenten, rowohlts monographien, herausgegeben von KuRT Kusenberg, Band 99, ohne Ort und Jahr, S. 63-66.

32 Ernst Haeckel, Die Radiolarien. Eine Monographie, Band I und II, Berlin 1862 und 1887.

${ }^{33}$ Report of the Scientific Results of the Voyage of H.M.S. Challenger during the Years 1872-1876. Ed. Sir Wyville Thomson and John Murray. Darin: A Summary of Scientific Results, First Part, London 1895. 
Damit ist die systematisch-entwicklungsgeschichtliche Phase der Planktonforschung abgeschlossen. Zwar werden noch einige neue Arten entdeckt, zwar sind noch einzelne Probleme in der Individualentwicklung einiger Spezies offen, doch entscheidende Neuigkeiten sind nicht mehr zu erwarten.

\section{III}

Der revolutionäre Impuls, der die dritte Periode der Planktonforschung einleitet, geht wiederum, wie schon in der zweiten Periode, von einem Mediziner aus. Wenn auch die Planktonmorphologie nahezu ausgeschöpft ist, so ist doch die Physiologie noch kaum in Angriff genommen worden, ja sogar die Fragestellungen stehen noch aus.

Hier ist es der Physiologe Victor Hensen (1835-1924), der in Kiel lehrte, also am Meer, und der ein neues Tor aufstieß und damit den Blick auf ein Forschungsgebiet so gewaltigen Ausmaßes freigab, daß noch heute nicht im mindesten von einer Lösung auch nur der brennendsten Probleme gesprochen werden kann. Das Stichwort für Hensens neue Arbeitsrichtung heißt: quantitative Untersuchungen.

Es sollen kurz die Entwicklungsgeschichte der Hensenschen Gedanken, seine für die angestrebten Lösungen entscheidende neue Methodik, einige seiner Arbeiten und Ergebnisse skizzenhaft erörtert werden. Sie sind nicht nur für die Planktonforschung, sondern für die gesamte Biologie von beispielhafter Bedeutung.

Victor Hensen ist aus der Stadt Schleswig gebürtig ${ }^{34}$. Sein Vaterhaus stand unmittelbar an den Ufern der Schlei, eines Meeresarms der Ostsee, so daß der junge Victor schon früh eine Beziehung zum Meer gewinnen konnte. $\mathrm{Da}$ das Meer im Geschwisterkreis Victors eine besondere Rolle spielte, läßt die Berufswahl einiger seiner Brüder erkennen: sie wurden Seefahrer. Victor aber tendierte zur Biologie und Medizin. Seine Studien führten ihn 1854 nach Würzburg. Dort gewann er seinen Kommilitonen CarL Semper (1832-1893) zum Freunde; Semper wurde später o. Professor für Zoologie in Würzburg. Die beiden Studenten machten ihre ersten meeresbiologischen

${ }^{34}$ Eine ausgedehnte Untersuchung über das Leben und Werk Victor Hensens vom Verfasser ist abgeschlossen und steht vor der Veröffentlichung (Diss. med. Kiel); siehe dortiges Literaturverzeichnis.

Wertvolles Material zur Geschichte der Planktonforschung, besonders auch die Kieler Schule berücksichtigend, bietet auch James Johnstone, Conditions of Life in the Sea. A Short Account of Quantitative Marine Biological Research, Cambridge 1908. 
Entdeckungen im Jahre 1856, wozu sie sich Material mit dem Müller-Netz aus der Kieler Förde fischten ${ }^{35}$. Hier liegt der Startpunkt für Hensens Planktonforschungen.

Hensen habilitierte sich im Jahre 1859 bald nach seiner Promotion zum Dr. med. et chir. in Kiel und wurde hier zum Prosektor am Anatomischen Institut ernannt. Schon 1864 bekam er einen Ruf auf den Lehrstuhl für Physiologie in Kiel und hatte ihn bis 1911 inne.

Aus der Fülle seiner Publikationen seien hier nur die meeresbiologischen herausgegriffen. Die ersten Veröffentlichungen dieser Richtung sind klassischer Art, gehören also der erörterten systematisch-entwicklungsgeschichtlichen Periode an. Sie wurden gefördert durch eine Studienreise nach Triest im Jahre 1863.

Nach seiner Wahl in den preußischen Landtag am 7. November 1867 trat Hensen sehr lebhaft für die Belange der Seefischerei ein und erreichte, daß 1870 die «Preußische Kommission zur Untersuchung der deutschen Meere» ins Leben gerufen wurde. Zu deren Sitz wurde Kiel, der Wirkungsort Hensens, bestimmt. Hensen war Mitglied der Kommission und Mitherausgeber des Publikationsorganes ${ }^{36}$.

Die Anfänge der quantitativen Forschungen in der Meeresbiologie haben ihre Wurzeln in der Fischereibiologie. Schon seit langem waren Kassandrarufe erschollen, es drohe eine Entvölkerung der Meere, wenn man die Fischerei weiter so intensiv betriebe wie bislang; andere sprachen dagegen von einem unerschöpflichen Reichtum der Meere. Hier wollte Hensen objektive Daten vorlegen, um die sinnlosen Spekulationen zu beenden. Seine Frage war: wie groß ist die Fischpopulation in einem abgegrenzten Areal. Die Methodik zur Lösung dieses Problems ist höchst originell. Da man die Fische nicht direkt zählen konnte, mußte eine indirekte Methode ersonnen werden.

Man wußte, daß die Eier einiger der wichtigsten Spezies pelagisch sind, also frei im Wasser schwimmen. Hensen nahm an, daß so kleine Teilchen wie Fischeier in einem so gleichförmigen Biotop, wie das Meer es darstellt, gleichmäßig dispergiert vorliegen müßten. Er folgerte daraus, daß die

35 Siehe Anm. 4, S. 48 und Victor Hensen, Über eine Brachiolaria des Kieler Hafens, Arch. Naturgeschichte (Troschels Arch.) 29 (1863) 242-246.

36 Jahresbericht der Kommission zur wissenschaftlichen Untersuchung der deutschen Meere in Kiel, Berlin (erster Jahresbericht Berlin 1873 bis sechster Jahresbericht Berlin 1893). Von 1894 an unter dem Titel: Wissenschaftliche Meeresuntersuchungen, herausgegeben von der Kommission etc. (siehe oben), N.F., Band 1 ff, Kiel/Leipzig 1894 ff. 
Untersuchung einiger Wassersäulen, die senkrecht vom Meeresboden bis zur Wasseroberfläche reichen, repräsentativ für ein jeweils größeres Areal sein müßte. Kennt man die Zahl der schwimmenden bzw. schwebenden Eier pro Areal und die Zahl der abgelegten Eier pro Fischweibchen, so kann man cum grano salis auf die Population an geschlechtsreifen Weibchen in diesem Areal schließen. Die Netzfänge der Berufsfischer geben über die Relation Weibchen zu Männchen Auskunft, so daß aus den vorhandenen Daten die Gesamtpopulation berechnet werden kann.

Um den Fang der pelagischen Fischeier zu bewerkstelligen, berechnete, konstruierte und erprobte Hensen ein Netz, das bei vertikalem Zuge exakt die Wassersäule durchfiltriert, die von der Netzöffnung aus den Wassermassen herausgeschnitten wird. Nach vielen Einzelerprobungen wurde die Methode auf zwei Expeditionen angewandt, die unter Hensens Leitung standen: die «Pommerania »-Expedition 1871 und die «Nordsee»-Expedition 1872.

Die weiteren Überlegungen waren folgende: der Fischbestand wird durch das Nahrungsangebot ganz wesentlich reguliert. Für die meisten Arten der wichtigsten Speisefische ist Plankton die Nahrung. Was lag näher, als die Planktonmasse aufgrund derselben Stichprobenüberlegungen quantitativ zu bestimmen, wie sie für die Fischeier Gültigkeit haben? Plankton war immer schon als lästiger Beifang bei den Fischeierfängen aufgetreten. Hensen baute sein Netz (noch heute übrigens als Hensen-Netz jedem Planktologen ein Begriff und noch heute im Gebrauch) ${ }^{37}$ für die spezifischen Belange der Planktonfischerei um und begann mit seinen Untersuchungen in der Kieler Förde. Auch hier ist entscheidend für die quantitative Methodik die Zählung der gefangenen Individuen, von denen man weiß, welche Wassermenge sie bevölkerten.

Die frühen Probefänge lieferten so ermutigende Ergebnisse, daß schon 1885 eine kleinere Plankton-Expedition in die Nordsee («Holsatia I») gewagt wurde, der bereits 1887 eine Ostsee-Expedition («Holsatia II») folgte. Im selben Jahr prägte Hensen den Begriff Plankton, wie früher schon erwähnt (siehe S. 196).

Die Krönung aller dieser Mühen brachte die große und berühmt gewordene «Plankton-Expedition» mit dem Dampfer «National», die unter Hensens Leitung stand. Ein bedeutender finanzieller Beitrag der Humboldt-

37 Z. B. Sir Alister C. Hardy, The Open Sea, Part I = The World of Plankton, Tafel XXIV, London 1956. 
Stiftung, eines Organs der preußischen Akademie der Wissenschaften in Berlin, dazu verschiedene Zuwendungen vom Kaiser, von Institutionen und Privatpersonen ermöglichten eine großangelegte Plankton-Expedition im Atlantischen Ozean vom 15. Juli bis zum 7. November 1889. Ihre vielfältigen und bedeutenden Ergebnisse, die in fünf Teilen zu je mehreren Quartbänden vorgelegt wurden, stammen aus der Feder einer großen Zahl zum Teil sehr bekannter Wissenschaftler. Hensen oblag die Herausgabe des Gesamtwerks ${ }^{38}$; der letzte Band erschien erst kurz nach seinem Tode.

Hensens bahnbrechende Arbeiten wurden in der wissenschaftlichen Welt lebhaft erörtert und auch heftig kritisiert. Wie fremd eingefleischten Morphologen die physiologische Denkweise Hensens war, zeigt sehr eindrucksvoll die Haeckelsche Polemik «Plankton-Studien ». ${ }^{39}$ Zunächst beweist Haeckel, daß er selber sich nicht die Mühe gemacht hat, Hensens Gedankengängen zu folgen; anschließend entwickelt er eigene Vorstellungen, die von den Ergebnissen Hensens und seiner Schule erheblich abweichen, und kommt schließlich zu dem Schluß (wenig zartfühlend, wie er mit wissenschaftlichen Kontrahenten umzuspringen pflegte), Hensen und seine Schule trieben baren Unsinn und verjubelten Staatsgelder auf eine höchst verwerfliche Weise. Es hat nicht der Verteidigungsschriften Hensens ${ }^{40}$ und seines Schülers ${ }^{41}$ Franz Schütt (1859-1921), seit 1895 o. Professor der Botanik in Greifswald, bedurft, um Hensen die vollste wissenschaftliche Anerkennung zu sichern.

Ein weiterführender Problemkreis sei hier noch kurz angeschnitten, dessen Grundlage Hensen gelegt hat, um dessen Lösung sich seine Schüler bemüht haben, und an dem noch heute intensiv gearbeitet wird. Hensen war vollkommen klar, daß die Bestimmung des Bestandes an Meeresorganismen, in der Hauptmasse repräsentiert durch Plankton, nur eine Station auf dem Wege zur Ermittlung der Produktion des Meeres an organischer Substanz sein konnte. Die Aufklärung der Urproduktion des Meeres, vergleichbar den Hektarerträgen des Landes, und die Erfassung des gesam-

38 Victor Hensen, Ergebnisse der in dem Atlantischen Ocean von Mitte Juli bis Anfang November 1889 ausgeführten Plankton-Expedition der Humboldt-Stiftung. Auf Grund von gemeinschaftlichen Untersuchungen einer Reihe von Fachforschern, herausgegeben von V.H., Kiel/Leipzig $1892 \mathrm{ff}$.

39 Ernst Haeckel, Plankton-Studien, Jena 1890.

40 Victor Hensen, Die Plankton-Expedition und Haeckel's Darwinismus, Kiel/Leipzig 1891.

41 Franz Schütt, Analytische Plankton-Studien, Kiel/Leipzig 1892. 
ten Stoffkreislaufs im Meer sah er als Fernziel der von ihm ins Leben gerufenen Forschungsrichtung an.

Mit Hensen ging eine Ära der Planktonforschung zu Ende, in der Mediziner eine so glanzvolle Rolle gespielt haben. Schon unter den Mitarbeitern und Schülern Hensens ist nicht ein einziger Mediziner mehr (das betrifft selbstverständlich nicht den Physiologen Hensen). Johannes Müller, Ernst Haeckel und Victor Hensen waren Biologen, die ihre Ausbildung in der medizinischen Fakultät genossen hatten. Mit dem Anwachsen des medizinischen Wissensgutes hat die reine Biologie im Medizinstudium und in der medizinischen Forschung an Gewicht verloren. Mit der fortschreitenden Spezialisierung und der verfeinerten Methodik ist der Fachbereich der Forscher, gesehen in der alten Perspektive, enger geworden. Der Übergang von der Medizin, speziell der Anatomie, über die vergleichende Anatomie zur Zoologie, was alles Johannes Müller, weniger schon dessen Schüler Koelliker noch als Einheit empfanden, wurde immer schwieriger. Carl Gegenbaur vertrat am Anfang seiner Laufbahn noch Zoologie und vergleichende Anatomie, während Ernst Haeckel sich von vornherein, obwohl von Hause aus Mediziner, die Zoologie auserkor. Die Wissensgebiete haben sich so weit voneinander entfernt, daß so entscheidende Impulse, wie die Mediziner Johannes Müller und Victor Hensen sie der Planktonforschung gaben, in den inzwischen emanzipierten Gebieten nicht mehr erwartet werden konnten.

Man muß zum Schluß die grundsätzliche Frage stellen, warum Mediziner, und nicht Zoologen, die Initiatoren und, im Anfang, die treibenden Kräfte der Planktonforschung gewesen sind. Zumindest eine Hypothese bietet sich uns als Antwort an: Die Zoologen waren fast völlig von den Arbeiten an der Systematik des Tierreichs absorbiert. Ihre Entdeckerfreude ließ sie auf der Suche nach neuen Arten bis in die entlegensten Winkel der Erde vordringen. Was sie suchten, waren (makroskopisch!) demonstrable Objekte.

Anders stellt sich die Lage bei den Medizinern dar. Zum einen hatte der Gedanke der Entwicklung (Evolution) bei ihnen schon sehr stark Platz gegriffen, angeregt durch das Studium der menschlichen Ontogenie; dies brachte sie sozusagen von selbst auf die kleinen und kleinsten Formen. Zum anderen waren sie im Gebrauch des Mikroskops geübter. Das eine machte sie zu den Pionieren der vergleichenden Anatomie, das andere zur Avantgarde der biologischen Mikroskopie. Beides zusammen bereitete den Boden für die Idee eines ihrer besten Köpfe, Joh. Müller, im Meerwasser mit dem Mikroskop nach «niederen » Formen zu suchen. 
Auch der Impuls für die quantitative Planktonforschung kam fast zwangsläufig aus der Medizin, denn hier hatte sich die Physiologie emanzipiert und war in kräftigem Aufschwunge begriffen, wozu die Ernennung der ersten Physiologen als Lehrstuhlinhaber in den fünfziger Jahren des 19. Jahrhunderts ganz erheblich beigetragen hat. Den Zoologen und Botanikern war die physiologische, besonders die quantifizierende Betrachtungsweise wesentlich unbekannt, daher zu fremd, als daß von ihnen Impulse in dieser Richtung zu erwarten gewesen wären. Somit dürfte die Rolle, die die Mediziner in der Frühphase der Planktonforschung gespielt haben, keineswegs eine zufällige, sondern geradezu eine «schicksalhafte» sein. 